\title{
Effectiveness comparison of various atrial fibrillation ablation methods in patients with common venous trunk
}

\author{
Edward Koźluk ${ }^{1, A-F}$, Dorota Zyśko 2,A,C-F, Agnieszka Piątkowska1,2,A,B,E,F, Marek Kiliszek 3,A,B,E,F, Piotr Lodziński 1,A,B,E,F, \\ Sylwia Małkowska ${ }^{1, A, B, E, F}$, Paweł Balsam ${ }^{1, A, B, E, F}$, Dariusz Rodkiewicz, ${ }^{1, A, B, E, F}$, Małgorzata Żukowska ${ }^{4, A, B, E, F}$, Grzegorz Opolski ${ }^{1, A, E, F}$ \\ ${ }^{1} 1^{\text {st }}$ Chair and Department of Cardiology, Medical University of Warsaw, Poland \\ ${ }^{2}$ Chair of Emergency Medicine, Wroclaw Medical University, Poland \\ ${ }^{3}$ Department of Cardiology and Internal Diseases, Military Institute of Medicine, Warszawa, Poland \\ ${ }^{4} 2^{\text {nd }}$ Faculty of Radiology, Medical University of Warsaw, Poland \\ A - research concept and design; B - collection and/or assembly of data; $\mathrm{C}$ - data analysis and interpretation; \\ $D$ - writing the article; $E$ - critical revision of the article; $F$ - final approval of the article
}

\author{
Address for correspondence \\ Dorota Zyśko \\ E-mail:dzysko@wp.pl \\ Funding sources \\ None declared \\ Conflict of interest \\ Edward Koźluk - proctor for ablation procedures \\ (Medtronic and Johnson \& Johnson) \\ Piotr Lodziński - proctor for ablation procedures \\ (Johnson \& Johnson) \\ Paweł Balsam - proctor for ablation procedures \\ (St. Jude Medical) \\ Marek Kiliszek - proctor for ablation procedures \\ (St. Jude Medical) \\ Other authors declare no conflict of interest
}

Received on March 22, 2017

Reviewed on June 25, 2017

Accepted on 0ctober 2, 2017

Published online on August 8, 2018

Cite as

Koźluk E, Zyśko D, Piątkowska A, et al. Effectiveness comparison of various atrial fibrillation ablation methods in patients with common venous trunk. Adv Clin Exp Med. 2019; 28(4):461-467. doi:10.17219/acem/78157

DOI

10.17219/acem/78157

\section{Copyright}

Copyright by Author(s)

This is an article distributed under the terms of the

Creative Commons Attribution Non-Commercial License

(http://creativecommons.org/licenses/by-nc-nd/4.0/)

\section{Abstract}

Background. Atrial fibrillation (AF) is a common clinical problem. The left atrium anatomy makes up a factor that may significantly affect the effectiveness of the AF ablation.

Objectives. The aim of the study was to evaluate a long-term effectiveness ablation in patients with common pulmonary vein trunk (CPVT) and AF.

Material and methods. The outcomes of 129 procedures in 95 patients with CPVT out of 1,475 procedures carried out in 1,150 patients with AF treated with ablation, were analyzed. Ablation with CART0 3 system (Johnson \& Johnson, New Brunswick, USA), cryoballoon, and the circular multipolar duty-cycled radiofrequency-based pulmonary vein ablation producer with catheter (PVAC) were considered as advanced methods. The following data was recorded for every patient: age, gender, AF duration and type, previous antiarrhythmic drugs, weight, height, any prior cardioversion, and comorbidities, including hypertension, diabetes, hypothyreosis, thyrotoxicosis, heart failure, and stroke/transient ischemic attack. The following anatomical factors were assessed: the presence of patent foramen ovale (PFO) and localization of the (PVT on the basis of venography or computed tomography (CT). In the $1^{\text {st }}$ year after ablation, 24-hour Holter monitoring was performed $3-5$ times, and the patients were encouraged to visit their doctor or an emergency department if a cardiac arrhythmia occurred. Long-term ablation effectiveness was assessed based on a telephone interview and patients' answers to the questionnaires including 12-lead electrocardiography (ECG).

Results. Sinus rhythm was maintained in 44 patients (43.6\%) after a median of 42 months (range: 12-120). A lower number of clinical factors (odds ratio $(O R)=0.09 ; 95 \%$ confidence interval $(95 \% \mathrm{Cl})=0.02-0.56$; $\mathrm{p}<0.01)$, and advanced ablation methods $(\mathrm{OR}=3.1 ; 95 \% \mathrm{Cl}=1.4-7.1 ; \mathrm{p}<0.01)$ were related to a better long-term effectiveness.

Conclusions. The long-term effectiveness of pulmonary vein (PV) isolation in patients with AF and CPVT is higher when advanced ablation techniques are used. Accumulation of clinical factors was found to be the most tremendous predictor of AF recurrence.

Key words: long-term outcome, atrial fibrillation, ablation, common trunk 


\section{Introduction}

Atrial fibrillation (AF) is a common clinical problem associated with the occurrence of sudden and non-sudden cardiac death, heart failure, stroke, an increased risk of hospitalizations, and decreased quality of life..$^{1-3}$

In the treatment of patients with symptomatic AF, isolation of pulmonary vein (PV) is recommended by the creation of circumferential lesions around the right and the left PV ostia. ${ }^{2-4}$ However, invasive procedures may lead to complications. ${ }^{4-7}$ Looking for predictors of atrial fibrillation relapse facilitates the process of qualifying the patient for the procedure, as well as the selection of the most effective methods to perform ablation. ${ }^{8-11}$

The left atrium anatomy makes up a factor that may significantly affect the effectiveness of the procedure. An unusual anatomy of PV, usually in the form of a common pulmonary vein trunk (CPVT), occurs in 13-29\% of patients. ${ }^{12,13}$ The vascular anomaly, which usually refers to single-sided PVs, is more common on the left side. ${ }^{4,13}$ The presence of the CPVT hampers PV isolation, the main reason for that possibly being a fact that systems for isolating the PVs have been developed for a typical PV anatomy, and their effectiveness might alter in the case of an unusual anatomy. The aim of the study was to evaluate the longterm effectiveness of different methods used to ablate/ isolate PV in patients to treat AF.

\section{Material and methods}

The study was designed as the retrospective analysis of the long-term results of ablation procedures performed in patients with AF and CPVT between 2003 and 2012.

Out of the group of 1,150 patients (1,475 procedures) with AF, 107 (9.3\%) had the CPVT recognized. Twelve patients did not appear at the follow-up after the $1^{\text {st }}$ procedure. Finally, the effectiveness of 129 procedures performed in 95 patients with the CPVT was subjected to analyses in the study. Table 1 shows the patients' characterization by the technique used for ablation. Among the studied population, 72 patients had 1 procedure only, 15 had 2 procedures, 6 had 3 procedures, 1 patient had 4 procedures, and 1 had 5 procedures. The mean number of ablation procedures amounted to 1.3 times per patient.

\section{Data collection}

The following data was recorded for every patient: age, gender, AF duration and type, previous antiarrhythmic drugs, weight, height, any prior cardioversion, and comorbidities including hypertension, diabetes, hypothyreosis, thyrotoxicosis, heart failure, as well as stroke/transient ischemic attack. The following echocardiographic data was recorded: left ventricle ejection fraction (EF) and left atrium diameter. Ejection fraction less than $50 \%$ was regarded as low EF. For every ablation an average fluoroscopy and procedure time were recorded.

During ablation, the following anatomical factors were assessed: the presence of patent foramen ovale (PFO; if it could be used to introduce the catheter into the left atrium), and left-side or right-side localization of the CPVT recognized on the basis of venography or computed tomography (CT). Furthermore, the method of ablation was recorded in each case and the nature of the procedure was referred to as the $1^{\text {st }}$ or the repeated procedure.

\section{Procedure details}

The objective of the procedure was to isolate all PVs. Other ablation regions were performed if the surgeon decided so. In some patients with high far-field potentials from the superior vena cava (VCS) or the inferior vena cava (VCI) ( $\mathrm{n}=50$ and $\mathrm{n}=4$, respectively), the vein was isolated, too. In patients with persistent AF, with a short distance between PV lines or with transformation of AF into an atypical atrial flutter, additional lines were performed. All lines were carried out using CARTO (Johnson \& Johnson, New Brunswick, USA) system, including left atrial roof $(n=10)$, posterior wall $(n=10)$ or the mitral isthmus $(n=8)$, right atrial isthmus $(n=13)$, coronary sinus $(\mathrm{n}=8)$, complex fractionated atrial electrogram (CFAE) $(\mathrm{n}=3)$, VCS VCI line $(\mathrm{n}=1)$, and septum $(\mathrm{n}=6)$.

In the presented study, fluoroscopy was used as the only imaging method in the subgroup of patients in whom Lasso electrode and ablation with 4-mm tip catheter were used for segmental PV isolation, using the method described by Haïssaguerre et al. ${ }^{14}$ The majority of the procedures performed by the use of Haïssaguerre's method were done with the LocaLisa system used to reduce fluoroscopy. Details of this technique were previously presented. ${ }^{14}$ The LocaLisa navigation system (Medtronic EP Systems, Minneapolis, USA) uses 3 low-amplitude, high-frequency current fields. The fields are generated in 3 axes over the patient's thorax and are dedicated to compute the position of an electrode in the thorax relative to a reference electrode. The procedure of segmental PV isolation using the LocaLisa navigation system was precisely described in the previous publication. ${ }^{15}$ Briefly, the Lasso catheter (Johnson \& Johnson, New Brunswick, USA) was located in every PV under the fluoroscopic guidance. The ablation procedure was performed by the use of the ablation catheter under the LocaLisa system and fluoroscopy. The PV isolation was proved by disappearance or dissociation of potential recorded from the Lasso catheter. ${ }^{15}$

CARTO XP (Biosense Webster, Johnson \& Johnson, New Brunswick, USA) visualized only the ablation catheter. This catheter was used to create the left atrial map. The next atrial PV linear isolation was performed based on the method presented by Pappone et al. ${ }^{16}$ Verification of the lines was done using the voltage map (reduction of the potentials in the isolated area $<0.1 \mathrm{mV}$ ). 
Table 1. Baseline demographics and clinical characteristics

\begin{tabular}{|c|c|c|c|c|c|c|c|}
\hline Characteristics & $\begin{array}{c}\text { Total group } \\
n=95\end{array}$ & $\begin{array}{l}\text { CARTO XP } \\
n=7\end{array}$ & $\begin{array}{c}\text { CARTO } 3 \\
n=6\end{array}$ & $\begin{array}{l}\text { Lasso } \\
\mathrm{n}=16\end{array}$ & $\begin{array}{l}\text { PVAC } \\
n=21\end{array}$ & $\begin{array}{l}\text { LocaLisa } \\
n=42\end{array}$ & $\begin{array}{c}\text { Cryoballoon } \\
n=3\end{array}$ \\
\hline Age [years] & $54.1 \pm 10.1$ & $54.6 \pm 7.4$ & $58.8 \pm 8.2$ & $53.3 \pm 10.9$ & $55.3 \pm 9.9$ & $52.9 \pm 10.8$ & $56.3 \pm 8.1$ \\
\hline Sinus rhythm maintenance, $\mathrm{n}$ [\%] & $31(32.4)$ & $2(28.6)$ & $2(33.3)$ & $2(12.5)$ & $10(47.6)$ & $13(31.0)$ & $2(66.7)$ \\
\hline Male gender, n [\%] & $59(62.1)$ & $5(71.4)$ & $3(50.0)$ & $8(50.0)$ & $10(47.6)$ & $31(73.8)$ & $2(66.7)$ \\
\hline Persistent AF, n [\%] & $23(24.2)$ & $6(85.7)$ & $3(50.0)$ & $1(6.3)^{\#}$ & $5(23.8)^{\#}$ & $7(16.7)^{\#}$ & $1(33.3)$ \\
\hline $\mathrm{LA}[\mathrm{mm}]$ & $42.4(6.6)$ & $45.9(3.7)$ & $44.0(2.8)$ & $41.8(5.5)$ & $43.1(4.9)$ & $41.9(8.2)$ & $35.7(1.2)$ \\
\hline EF [\%] & $60.1(6.5)$ & $58.1(8.3)$ & $57.3(6.7)$ & $61.2(4.9)$ & $60.1(8.1)$ & $59.9(7.0)$ & $66.3(2.3)$ \\
\hline $\mathrm{CHA}_{2} \mathrm{DS}_{2}-$ VASc median (IQR) & $1(1-2)$ & $1(1-2)$ & $1(1-2)$ & $1(1-2)$ & $1(1-2)$ & $1(0-2)$ & $0(0-2)$ \\
\hline Low EF, n [\%] & $4(4.2)$ & $1(14.3)$ & $0(0)$ & $0(0)$ & $1(4.8)$ & $2(4.8)$ & $0(0)$ \\
\hline CPVT right or bilateral, n [\%] & $72(75.8)$ & $6(85.7)$ & $1(16.7)$ & $11(66.8)$ & $17(81)$ & $35(83.3)$ & $2(66.7)$ \\
\hline Ablation in VCS ostium, n [\%] & $41(43.2)$ & $2(28.6)$ & $0(0)$ & $8(50.0)$ & $8(38.1)$ & $23(54.8)$ & $0(0)$ \\
\hline $\mathrm{FOA}, \mathrm{n}[\%]$ & $12(12.6)$ & $0(0)$ & $0(0)$ & $0(0)$ & $5(23.8)$ & $7(16.7)$ & $0(0)$ \\
\hline History of the electric cardioversion, $\mathrm{n}$ [\%] & $48(50.5)$ & $6(85.7)$ & $5(83.3)$ & $7(43.08)$ & $11(52.4)$ & $18(42.9)$ & $1(33.3)$ \\
\hline History of the thyroid diseases, n [\%] & $21(22.1)$ & $2(28.6)$ & $1(16.7)$ & $4(25.0)$ & $4(19.0)$ & $10(23.8)$ & $0(0)$ \\
\hline Hypertension, n [\%] & $55(57.9)$ & $5(71.4)$ & $4(66.7)$ & $11(68.8)$ & $11(52.4)$ & $23(54.8)$ & $1(33.3)$ \\
\hline Diabetes, n [\%] & $8(8.4)$ & $0(0)$ & $2(33.3)$ & $2(12.5)$ & $2(9.5)$ & $2(4.8)$ & $0(0)$ \\
\hline COPD, n [\%] & $3(3.2)$ & $1(14.3)$ & $1(16.7)$ & $0(0)$ & $0(0)$ & $1(2.4)$ & $0(0)$ \\
\hline Previous stroke, n [\%] & $7(7.4)$ & $1(14.3)$ & $0(0)$ & $1(6.3)$ & $2(9.5)$ & $3(7.1)$ & $0(0)$ \\
\hline Number of clinical factors, mean $\pm S D$ & $1.8 \pm 1.3$ & $3 \pm 0.8$ & $2.7 \pm 1.4$ & $1.7 \pm 1.3$ & $1.9 \pm 1.4$ & $1.6 \pm 1.1$ & $1.0 \pm 1.0$ \\
\hline Follow up [months], mean $\pm S D$ & $48.7 \pm 25.7$ & $44.7 \pm 21.1^{*}$ & $19.0 \pm 4.1^{* \$}$ & $82.9 \pm 24.2^{\$}$ & $29.4 \pm 6.0^{* \$}$ & $51.6 \pm 19.7^{*}$ & $30.7 \pm 13.7^{*}$ \\
\hline Procedure duration [h] & $2.4 \pm 0.8$ & $3.7 \pm 0.8$ & $2.5 \pm 0.5^{\#}$ & $2.5 \pm 0.6^{\#}$ & $2.1 \pm 0.6^{\#}$ & $2.1 \pm 0.6^{\#}$ & $2.8 \pm 0.4$ \\
\hline Fluoroscopy duration [min] & $20.7 \pm 12.4$ & $14.2 \pm 9.3^{*}$ & $15.7 \pm 5.7^{*}$ & $37.5 \pm 19.0$ & $18.7 \pm 6.9^{*}$ & $19.7 \pm 11.0^{*}$ & $28.9 \pm 10.5$ \\
\hline
\end{tabular}

AF - atrial fibrillation; LA - left atrium; EF - ejection fraction; COPD - chronic obstructive pulmonary disease; FOA - foramen ovale apertum; VCS - vena cava superior; PVAC - pulmonary vein ablation producer with catheter; CPVT - common pulmonary vein trunk; IQR - interquartile range; SD - standard deviation; * $p<0.001$ vs Lasso; ${ }^{*} p<0.001$ vs CARTO XP; $\$ p<0.001$ vs LocaLisa.

CARTO 3 (Biosense Webster, Johnson \& Johnson, New Brunswick, USA) system is an upgraded version, allowing a more accurate visualization of the cardiac anatomy (e.g., to perform a quick anatomical map) and enabling the visualization of all catheters used. This PV isolation verification technology was used for the Lasso catheter.

Ablation with PV ablation producer with catheter (PVAC) (Medtronic, Carlsbad, USA) was described in most detail in the previous publication. ${ }^{17}$ Shortly after a PV selective angiography a circular decapolar catheter is advanced over wire positioned selectively in every PV. After several radio frequency $(\mathrm{RF})$ applications over all electrode pairs, the PVAC was rotated around the PV ostium to ensure a complete isolation of the vein. During the ablation of large common ostia, electrode pairs with suboptimal contact to the atrial tissue were deactivated, preventing ineffective energy delivery. ${ }^{17}$

Cryoballoon ablation methods were described in the previous publications. ${ }^{18}$ Shortly after an initial mapping of PV using the Lasso catheter, the cryoballoon (Arctic Front - Cryocath, Medtronic, Mineapolis, USA) was introduced into the left atrium and positioned at PV ostia. Contact of the balloon with the ostial tissue was checked by contrast administration and temperature inside the balloon during cryoapplication. At every PV ostium 2 cryoapplications with good contact were performed. Subsequently, remapping of cryoapplications in all veins was performed with Lasso catheters. In the absence of PV potentials, the procedure was finished; otherwise, next cryoapplications were performed. ${ }^{18}$

Ablations with CARTO 3 system, cryoballoon, and PVAC catheter were considered as advanced methods, whereas those with CARTO XP, Lasso guided alone or with LocaLisa system were regarded as reference methods, potentially less effective because of hindered adaptation to the changed anatomy.

\section{Follow-up}

The details of follow-up procedures were presented elsewhere. ${ }^{19}$ Briefly, in the $1^{\text {st }}$ year after ablation, 24-h Holter monitoring was performed 3-5 times, and the patients were encouraged to visit their doctor or an emergency department if a cardiac arrhythmia occurred. The procedure was assessed as ineffective when an event of AF lasting at least $30 \mathrm{~s}$ was documented later than after 3 months of a blanking period following the procedure. ${ }^{8}$ Long-term ablation effectiveness was assessed based on the telephone interview and patients' answers to the questionnaires, including 12-lead electrocardiography (ECG). The gathered data was further 
supplemented with the data from cardiology outpatient clinics and the results of the Holter monitoring. Had there been more than 1 ablation, all treatments preceding the last treatment (also in patients with whom the contact was lost) would have been considered ineffective.

Informed consent was obtained from all patients. The study was approved by the Bioethical Commission (Wroclaw Medical University, Poland).

Continuous variables were expressed as mean and standard deviation (SD) and compared with analysis of variance (ANOVA) test with Bonferroni correction for a multiple comparison. Categorical variables were presented as numbers and percentages, and $X^{2}$ test together with Fisher's exact test were both used to assess the differences.

The $\mathrm{CHA}_{2} \mathrm{DS}_{2}$-VASc score was calculated for each patient. The number of clinical factors was calculated for each patient as a sum of points. One point was given for the presence of each diagnosis in the patients' medical history: age above 65 years, age above 75 years, hypertension, diabetes mellitus, previous stroke, previous myocardial infarction, congestive heart failure, the presence of the permanent character of AF, previous electric cardioversion, thyroid diseases, and chronic obstructive pulmonary disease (COPD).

The logistic regression analysis was performed to find association between the AF recurrence, the advanced method of ablation and the number of clinical factors which may predict AF relapse.
The classification and regression tree analysis were used to find associations among the long-term outcome, the method of ablation and the clinical predictors of the AF recurrence. Specificity, sensitivity, and positive and negative predictive values for each model were calculated. The classification and regression tree analysis constitute non-parametric methods to establish predictors of the studied end-point. These methods were described by Breiman et al. and they consist in recursive partitioning of the study group for each possible point to find those dividing the entire group into subgroups which are differentiated the most regarding the evaluated events. ${ }^{19,20}$ The classification tree is a graphical method used for presenting the classification rules. The number in the upper right corner of the rectangle is an information about which subgroup it was included to, based on the classification rules. In the case of the present study " 0 " means maintaining sinus rhythm and " 1 " is the AF recurrence. The number of factors associated with the AF recurrence was assessed for every patient.

\section{Results}

Demographics, clinical characteristics within the study group and subgroups selected on the basis of the $1^{\text {st }}$ procedure performed were presented in Table 1 . Table 2 pictures the data regarding demographics and clinical

Table 2. Demographics and clinical characteristics in groups selected on the basis of the procedure performer

\begin{tabular}{|c|c|c|c|c|c|c|c|}
\hline Characteristics & $\begin{array}{l}\text { Total group } \\
n=129\end{array}$ & $\begin{array}{c}\text { CARTO XP } \\
n=17\end{array}$ & $\begin{array}{c}\text { CARTO } 3 \\
n=11\end{array}$ & $\begin{array}{l}\text { Lasso } \\
\mathrm{n}=19\end{array}$ & $\begin{array}{l}\text { PVAC } \\
n=24\end{array}$ & $\begin{array}{l}\text { LocaLisa } \\
n=52\end{array}$ & $\begin{array}{c}\text { Cryoballoon } \\
n=6\end{array}$ \\
\hline Age [years] & $54.7 \pm 10.2$ & $57.5 \pm 6.8$ & $59.9 \pm 6.6$ & $54.2 \pm 10.4$ & $54.4 \pm 10.8$ & $52.5 \pm 11.1$ & $59.8 \pm 7.1$ \\
\hline Sinus rhythm maintenance, $\mathrm{n}$ [\%] & $44(34.1)$ & $4(23.5)$ & $5(45.5)$ & $2(10.5)$ & $12(50.0)$ & $16(30.8)$ & $5(83.3)$ \\
\hline Male gender, n [\%] & $84(65.1)$ & $11(64.7)$ & $7(63.6)$ & $9(47.4)$ & $13(54.2)$ & $39(75.0)$ & $5(83.3)$ \\
\hline Persistent AF, n [\%] & $27(20.9)$ & $8(47.1)$ & $3(27.3)$ & $1(5.3)$ & $5(20.8)$ & $9(17.3)$ & $1(16.7)$ \\
\hline CPVT right or bilateral, $\mathrm{n}[\%]$ & $96(74.4)$ & $12(70.6)$ & $5(45.5)$ & $14(73.7)$ & $19(79.2)$ & $41(78.8)$ & $5(83.3)$ \\
\hline Ablation in VCS ostium, n [\%] & $50(38.8)$ & $2(11.8)$ & $0(0)$ & $9(47.4)$ & $10(41.7)$ & $29(55.8)$ & $0(0)$ \\
\hline FOA, n [\%] & $14(10.9)$ & $0(0)$ & $1(9.1)$ & $0(0)$ & $5(20.8)$ & $8(15.4)$ & $0(0)$ \\
\hline History of the electric cardioversion, n [\%] & $66(51.2)$ & $12(70.6)$ & $6(54.5)$ & $9(47.4)$ & $13(54.2)$ & $23(44.2)$ & $3(50.0)$ \\
\hline History of the thyroid diseases, n [\%] & $30(23.3)$ & $5(29.4)$ & $5(45.5)$ & $4(21.1)$ & $4(16.7)$ & $12(23.1)$ & $0(0)$ \\
\hline Diabetes, n [\%] & $10(7.8)$ & $0(0)$ & $3(27.3)$ & $2(10.5)$ & $2(8.3)$ & $3(5.8)$ & $0(0)$ \\
\hline COPD, n [\%] & $4(3.1)$ & $2(11.8)$ & $1(9.1)$ & $0(0)$ & $0(0)$ & $1(1.9)$ & $0(0)$ \\
\hline Previous stroke, n [\%] & $10(7.8)$ & $3(17.6)$ & $0(0)$ & $1(5.3)$ & $2(8.3)$ & $4(7.7)$ & $0(0)$ \\
\hline Number of clinical factors, mean $\pm S D$ & $1.8 \pm 1.2$ & $2.5 \pm 1.2$ & $2.2 \pm 1.7$ & $1.6 \pm 1.2$ & $1.8 \pm 1.3$ & $1.7 \pm 1.1$ & $1.0 \pm 0.9$ \\
\hline Follow up [months], mean $\pm S D$ & $48.7 \pm 10.1$ & $51.5 \pm 26.3^{*}$ & $21.0 \pm 9.0^{* \# \$}$ & $84.3 \pm 24.2$ & $29.5 \pm 5.7^{* \# \$}$ & $52.2 \pm 18.9^{*}$ & $25.5 \pm 12.8^{\# \$ *}$ \\
\hline Procedure duration [h] & $2.4 \pm 0.8$ & $3.6 \pm 1.0$ & $2.5 \pm 0.5^{\#}$ & $2.4 \pm 0.6^{\#}$ & $2.2 \pm 1.1^{\#}$ & $2.1 \pm 0.6^{\#}$ & $2.8 \pm 0.4^{\#}$ \\
\hline Fluoroscopy duration [min] & $21.4 \pm 13.2$ & $14.6 \pm 9.5^{*}$ & $15.7 \pm 9.5^{*}$ & $35.5 \pm 12.4$ & $18.7 \pm 6.9^{*}$ & $19.0 \pm 11.1^{*}$ & $28.9 \pm 10.5^{\#}$ \\
\hline
\end{tabular}

AF - atrial fibrillation; LA - left atrium; EF - ejection fraction; COPD - chronic obstructive pulmonary disease; FOA - foramen ovale apertum; VCS - vena cava superior; PVAC - pulmonary vein ablation producer with catheter; CPVT - common pulmonary vein trunk; SD - standard deviation; ${ }^{*} \mathrm{P}<0.001$ vS Lasso; ${ }^{p}<0.001$ vs CARTO XP; $\$ p<0.001$ vs LocaLisa. 
characteristics in groups selected on the basis of the procedure performed. The subgroups did not differ in terms of age and gender distribution. The number of clinical factors associated with AF relapse was significantly greater in the group treated with CARTO XP system than with LocaLisa. Procedure times were the longest during procedures with CARTO XP.

\section{Procedure safety}

Two events of transient phrenic nerve palsy during cryoablation were observed, while in 1 patient a mild stenosis of PVs occurred during the long-term followup (from $16 \times 14 \mathrm{~mm}$ before the procedure to $9 \times 6 \mathrm{~mm}$ thereafter). Patient with the PV stenosis presented with hemoptysis and tussis. These symptoms subsided after a few months. This patient had the ablation performed with LocaLisa.

No significant differences were found with reference to the overall complication rate.

\section{Follow-up}

During the follow-up of a median of 42 months (range 12-120 months), the sinus rhythm was maintained in 44 patients (43.6\%). Among 129 procedures, 85 procedures (65.9\%) were assessed as ineffective.

\section{Clinical variables associated with atrial fibrillation recurrence}

Thyroid diseases history was more prevalent in the group with AF recurrence than in the group without AF recurrence $(29.7 \%$ vs $6.5 \%$; $<<0.02$, respectively). No differences in the occurrence of other clinical variables related to the AF recurrence were observed. However, it is worthwhile to mention that the number of clinical factors associated with recurring AF was significantly lower in patients without AF recurrence than in those with AF recurrence $(1.4 \pm 1.2$ vs $2.0 \pm 1.2 ; \mathrm{p}<0.01$, respectively).

\section{Multivariate analysis}

The logistic regression analysis revealed that a lower number of clinical factors (odds ratio $(\mathrm{OR})=0.09,95 \%$ confidence interval $(95 \% \mathrm{CI})=0.02-0.56 ; \mathrm{p}<0.01)$ and advanced ablation methods $(\mathrm{OR}=3.1,95 \% \mathrm{CI}=1.4-7.1$; $\mathrm{p}<0.01)$ were related to better long-term effectiveness of the given procedure.

The classification and regression analysis confirmed the significance of the advanced methods for good outcome, whereas the low EF and the presence of PFO were related to the AF recurrence (Fig. 1), with the specificity of that model being 0.85 , sensitivity 0.90 , and positive and negative predictive values being 0.87 and 0.69 , respectively.

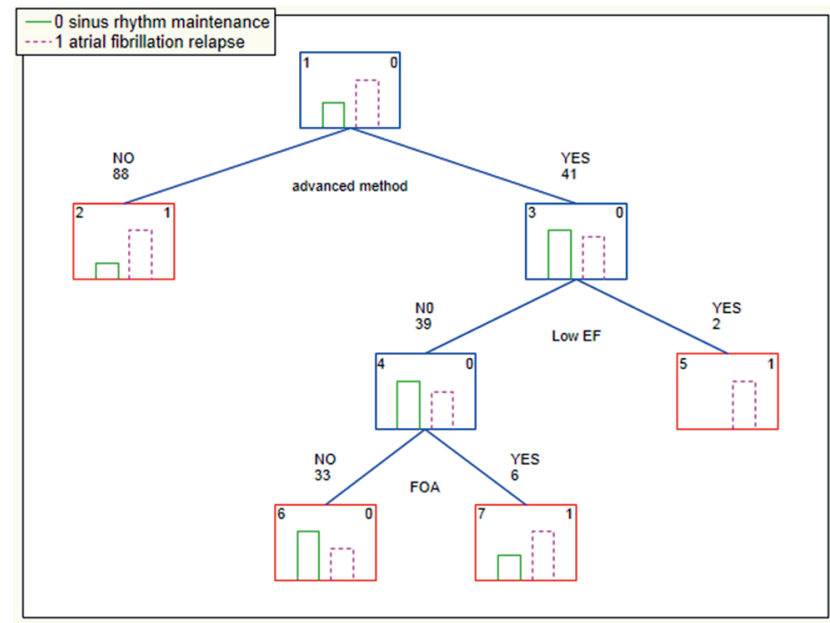

Fig. 1. Factors related to sinus rhythm maintenance

\section{Discussion}

The procedures of the PVs isolation are currently among the most effective therapeutic options for the AF treatment. The ablation effect on AF might be attributed to various mechanisms, including the elimination of the trigger, modification of the arrhythmogenic substrate, interruption of crucial pathways of conduction or rotors, or atrial denervation.

The PV isolation techniques differ with regards to the energy source (radiofrequency or cryoenergy), mapping system (electrophysiological or electroanatomical) and electrode shape (focal, ring or balloon). The effectiveness of these techniques may vary in the settings of the CPVT and normal anatomy.

Difficult anatomy of venoatrial junction may impede the effective implementation of the ablation procedure. Common pulmonary vein trunk is a quite frequently encountered variant of the PV anatomy. The size and shape of the veins ostia may be of particular importance for some of the techniques, whilst others may be more resistant to the associated difficulties.

Recent randomized studies comparing various methods of ablation techniques were performed in the setting of 4 independent PVs and demonstrated that the simplified strategy for PV cryoablation is inferior to PV isolation using open-irrigated radiofrequency catheters with electrophysiological and electroanatomical guidance. ${ }^{21}$ It was also observed that a complete PV conduction block is critical to the success of AF ablation. Malmborg et al. reported that either cryoballoon and/or the circular multipolar duty-cycled radiofrequency-based PVAC proved to be comparably effective and safe in achieving acute PV isolation. ${ }^{22}$ Other researchers have determined that among different procedures of catheter ablation there are no significant differences in the success rate between the 2 single procedures. Notwithstanding, the success rates are higher for combined methods than for single methods. ${ }^{23}$ 
The accuracy of the spatial localization by different advanced electroanatomical mapping systems seems to be comparable; however, in the case of the CPVT other factors may appear to be more important.

Furthermore, there are no established strategies for redo procedures after PV isolation. In the presented study, it was found that the advanced techniques are more effective than the older ones, regardless of whether used during the $1^{\text {st }}$ or the repeated procedure. Contrary to the presented findings, Pokushalov et al. reported that when patients require a redo PV isolation ablation procedure for the recurrent paroxysmal AF, RF appears to be the preferred method relative to cryoablation. ${ }^{24}$ However, the number of cryoballoon isolations in the presented study was too low to obtain conclusive results.

The clinical factors analyzed in the presented study overlap, but are not limited to, the parameters used in the $\mathrm{CHA}_{2} \mathrm{DS}_{2}-\mathrm{VASc}$ scale. Other factors assigned to the clinical picture include the following: the permanent character of AF, previous electric cardioversion, thyroid diseases, and COPD. ${ }^{9}$

The importance of clinical factors in predicting the longterm outcome of ablation procedure was confirmed in the study. The increase of the number of clinical factors associated with the reduced effectiveness of long-term ablation was associated with an increased risk of AF recurrence by $30 \%$. The classification and regression tree analysis revealed that thyroid disease constituted the most important clinical factor. It is well known that thyroid diseases often have the $1^{\text {st }}$ clinical manifestation as cardiac supraventricular arrhythmias. Thyroid hormones directly regulate the metabolism of myocardial cells, and thyroid diseases induce a diffuse atrial architecture disruption. This may predispose to ablation ineffectiveness. ${ }^{25-27}$ The PV isolation procedure is targeted at removing the trigger off the PVs rather than treating the whole diseased atrial myocardium which constitutes the substrate for AF.

The presence of thyroid diseases may also represent the adverse effect of amiodarone therapy. Patients with a greater tendency to relapse could have been treated with amiodarone, which in turn could have induced thyroid diseases, further promoting AF.

The treatment with amiodarone may lead to thyroid dysfunction, which then reduces the effectiveness of ablation. Therefore, it should be considered whether in patients with paroxysmal AF, ablation should not take precedence over pharmacological treatment. A major problem in iatrogenic hyperthyroidismcan particularlybethelongtimetogeteuthyroid.

Atrial fibrillation is a frequent rhythm in heart failure patients. It may exacerbate heart failure, which in turn may increase the susceptibility to AF occurrence. Heart failure is also a predictor of AF recurrence after ablation procedure, which was also determined in the presented study. An explanation for this observation may be the occurrence of changes in the myocardium of heart failure patients, which lead to the transformation of arrhythmia from trigger-driven to substrate-mediated. The risk of AF recurrence after catheter ablation is also increased in another setting of the atrial myocardium damage as sick sinus syndrome. ${ }^{28}$

Such anatomical factors as the localization of the CPVT and the presence of PFO were also included in the analysis because in the previous analysis we had found that these factors may have an impact on the effectiveness of the performed procedure. In the current analysis, contrary to the one performed earlier, only PFO presence was related to the lower effectiveness. The ablation methods used may have various effectiveness levels in the case of the right-side CPVT, which made it difficult to show the adverse significance of the localization of the CPVT.

There are no established strategies for the redo procedures after the PV isolation. The presented study indicates the higher effectiveness of the advanced methods both in the $1^{\text {st }}$ and in the redo procedures.

\section{Limitations}

This study is subjected to several limitations. Firstly, the follow-up duration varies among the patients' groups based on methods that were used. However, even after taking into account a 5\% annual increase in the risk of recurrence, the presented results indicate the higher effectiveness of newer ablation techniques. ${ }^{29}$ Secondly, the limited number of patients and a single-center study design may produce a bias, thus these results may not be generalized.

The methods used for a long-term follow-up after AF catheter ablation are not as accurate as continuous ECG monitoring. ${ }^{30}$ However, these methods allow us to find recurrences in the $2 / 3$ of patients, although the immediate success rate was comparable with other studies.

Institutional experience over nearly 10 years may improve significantly; however, the total number of procedures was high, so the effect of a learning curve could affect only a small percentage of cases.

\section{Conclusions}

The long-term effectiveness of PV isolation in patients with AF and CPVT was higher when advanced ablation techniques were used.

The same group of ablation methods is more effective in treatment, regardless of whether it is applied during the $1^{\text {st }}$ or the redo procedure.

Accumulation of the clinical risk factors was found to be the most tremendous predictor of AF recurrence, and this finding could be helpful in selecting patients with AF for an appropriate therapeutic strategy.

Thyroid disease seems to be the most significant clinical factor related to the AF recurrence after ablation.

Computed tomography scan before PV isolation and the performance of the ablation by experienced operator in the presence of CPVT should be recommended. 


\section{References}

1. Chen LY, Sotoodehnia N, Bůžková P, et al. Atrial fibrillation and the risk of sudden cardiac death: The atherosclerosis risk in communities study and cardiovascular health study. JAMA Intern Med. 2013;173(1):29-35.

2. Camm AJ, Lip GY, De Caterina R, et al. 2012 focused update of the ESC Guidelines for the management of atrial fibrillation: An update of the 2010 ESC Guidelines for the management of atrial fibrillation. Developed with the special contribution of the European Heart Rhythm Association. Eur Heart J. 2012;33(21):2719-2747.

3. European Heart Rhythm Association; European Association for CardioThoracic Surgery; Camm AJ, Kirchhof P, Lip GY, et al. Committee for Practice Guidelines. Guidelines for the management of atrial fibrillation: The Task Force for the Management of Atrial Fibrillation of the European Society of Cardiology (ESC). Europace. 2010;12:1360-1420.

4. Calkins H, Kuck KH, Cappato R, et al. 2012 HRS/EHRA/ECAS Expert Consensus Statement on Catheter and Surgical Ablation of Atrial Fibrillation: Recommendations for patient selection, procedural techniques, patient management and follow-up, definitions, endpoints, and research trial design. Europace. 2012;14:528-606.

5. Sotomi $\mathrm{Y}$, Inoue $\mathrm{K}$, Ito $\mathrm{N}$, et al. Incidence and risk factors for very late recurrence of atrial fibrillation after radiofrequency catheter ablation. Europace. 2013;15:1581-1586.

6. Cai L, Yin Y, Ling Z, et al. Predictors of late recurrence of atrial fibrillation after catheter ablation. Int J Cardiol. 2013;164(1):82-87.

7. Van Brabandt $H$, Neyt M, Devos C. Effectiveness of catheter ablation of atrial fibrillation in Belgian practice: A cohort analysis on administrative data. Europace. 2013;15:663-668.

8. Fein AS, Shvilkin A, Shah D, et al. Treatment of obstructive sleep apnea reduces the risk of atrial fibrillation recurrence following catheter ablation. J Am Coll Cardiol. 2013;62(4):300-305.

9. Chao TF, Cheng CC, Lin WS, et al. Associations among the CHADS(2) score, atrial substrate properties, and outcome of catheter ablation in patients with paroxysmal atrial fibrillation. Heart Rhythm. 2011;8(8):1155-1159.

10. Beukema RP, Beukema WP, Smit JJ, et al. Efficacy of multi-electrode duty-cycled radiofrequency ablation for pulmonary vein disconnection in patients with paroxysmal and persistent atrial fibrillation. Europace. 2010;12:502-507.

11. Monteiro MM, Saraiva C, Castelo Branco J, Cavaco D, Adragão P. Characterization of pulmonary vein morphology using multi-detector row CT study prior to radiofrequency ablation for atrial fibrillation. Rev Port Cardiol. 2009;28(5):545-559.

12. den Uijl DW, Tops LF, Delgado V, et al. Effect of pulmonary vein anatomy and left atrial dimensions on outcome of circumferential radiofrequency catheter ablation for atrial fibrillation. Am J Cardiol. 2011;107(2):243-249.

13. Sohns C, Sohns JM, Bergau L, et al. Pulmonary vein anatomy predicts freedom from atrial fibrillation using remote magnetic navigation for circumferential pulmonary vein ablation. Europace. 2013;15:1136-1142.

14. Haïssaguerre $M$, Macle $L$, Jaïs $P$, et al. Electrophysiologically guided pulmonary vein isolation during sustained atrial fibrillation. $J$ Cardiovasc Electrophysiol. 2003;14(3):255-260.
15. KoźlukE, Gawrysiak M, Lodziński P, et al. The LocaLisa system as the key to shortening the procedure duration and fluoroscopy time during ablation of atrial fibrillation. Kardiol Pol. 2008;66(6):624-629 (discussion 630-631).

16. Pappone C, Rosanio S, Oreto G, et al. Circumferential radiofrequency ablation of pulmonary vein ostia: A new anatomic approach for curing atrial fibrillation. Circulation. 2000;102(21):2619-2628.

17. Koźluk E, Balsam P, Peller M, et al. Efficacy of multi-electrode dutycycled radiofrequency ablation in patients with paroxysmal and persistent atrial fibrillation. Cardiol J. 2013;20:618-625.

18. Koźluk E, Gaj S, Piatkowska A, et al. Evaluation of safety and the success rate of cryoballoon ablation of the pulmonary vein ostia in patients with atrial fibrillation: A preliminary report. Kardiol Pol. 2010;68(2):175-180.

19. Koźluk E, Zyśko D, Piątkowska A, et al. Clinical and anatomical models of atrial fibrillation ablation in patients with common pulmonary vein trunk: Preliminary report. Pol Prz Kardiol. 2013;15(4):241-249.

20. Breiman L, Friedman JH, Olshen A, Stone CJ. Classification and Regression Trees. Belmont, CA: Wadsworth; 1984.

21. Pérez-Castellano N, Fernández-Cavazos R, Moreno J, et al. The COR trial: A randomized study with continuous rhythm monitoring to compare the efficacy of cryoenergy and radiofrequency for pulmonary vein isolation. Heart Rhythm. 2014;11(1):8-14.

22. Malmborg H, Lönnerholm S, Blomström P, Blomström-Lundqvist $C$. Ablation of atrial fibrillation with cryoballoon or duty-cycled radiofrequency pulmonary vein ablation catheter: $A$ randomized controlled study comparing the clinical outcome and safety. The AF-COR study. Europace. 2013;15:1567-1573.

23. Lin G, Lu HH, Shen Y, Huang JF, Shi LS, Guo YN. Meta-analysis of the therapeutic effects of various methods for the treatment of chronic atrial fibrillation. Exp Ther Med. 2013;6(2):489-496.

24. Pokushalov E, Romanov A, Artyomenko S, et al. Cryoballoon versus radiofrequency for pulmonary vein re-isolation after a failed initial ablation procedure in patients with paroxysmal atrial fibrillation. $J$ Cardiovasc Electrophysiol. 2013;24(3):274-279.

25. Tang RB, Liu DL, Dong JZ, et al. High-normal thyroid function and risk of recurrence of atrial fibrillation after catheter ablation. Circ $J$. 2010;74:1316-1321.

26. Mikhaylov EN, Orshanskaya VS, Lebedev AD, Szili-Torok T, Lebedev DS. Catheter ablation of paroxysmal atrial fibrillation in patients with previous amiodarone-induced hyperthyroidism: A case-control study. J Cardiovasc Electrophysiol. 2013;24(8):888-893.

27. Zimetbaum P. Amiodarone for atrial fibrillation. N Engl J Med. 2007;356(9):935-941.

28. Wu JT, Dong JZ, Sang CH, Tang RB, Li XH, Ma CS. Efficacy of catheter ablation for atrial fibrillation in patients with a permanent pacemaker for sick sinus syndrome. Intern Med. 2013;52(20):2305-2310.

29. Weerasooriya R, Khairy $P$, Litalien J, et al. Catheter ablation for atrial fibrillation: Are results maintained at 5 years of follow-up? J Am Coll Cardiol. 2011;57(2):160-166.

30. Tondo C, Tritto M, Landolina M, et al. Rhythm-symptom correlation in patients on continuous monitoring after catheter ablation of atrial fibrillation. J Cardiovasc Electrophysiol. 2014;25(2):154-160. 